\title{
EXEMPLES DE PÉDIATRIE SOCIALE APPLIQUÉE EN SUISSE
}

Kurt Albermann, Saskia von Overbeck Ottino, Tina Huber-Gieseke, Dorothea Schultz

Traduction: Rudolf Schlaepfer

Kurt Albermann

Saskia von

Overbeck Ottino

Tina Huber-Gieseke

Dorothea Schultz

https://doi.org/ 10.35190/f2021.4.7
La pédiatrie sociale se pratique en Suisse sous différentes formes, là où des lacunes dans la prise en charge biopsychosociale sont constatées et des acteurs locaux cherchent et créent des solutions en collaboration interdisciplinaire. À notre demande sont présentées ici, à titre d'exemple, quatre offres: un centre de pédiatrie sociale dans une clinique pédiatrique, une approche interdisciplinaire pour la santé psychique des enfants, adolescents et familles réfugiés, un projet visant la prise en charge globale de la santé et l'accueil d'enfants en âge scolaire issus de la migration, ainsi qu'une démarche favorisant l'intégration scolaire d'élèves avec une maladie chronique. La liste pourrait certainement encore être allongée et peut-être que ces exemples vont créer des vocations...

\section{Centre de pédiatrie sociale Winterthour (SPZ)}

Dr Kurt Albermann, Centre de pédiatrie sociale, Département de pédiatrie, Hôpital cantonal Winterthour, kurt.albermann@ksw.ch

Le Centre de pédiatrie sociale (SPZ) à Winterthour a été créé en 2003 et fait partie du Département de pédiatrie de l'Hôpital cantonal. Au SPZ existent des possibilités d'investigation, de traitement et de conseil pour enfants et adolescents dans les domaines de la pédopsychiatrie et de la psychosomatique, pédiatrie du développement et neuropédiatrie. En outre le SPZ propose depuis 2013, à la demande de la Direction de l'enseignement du canton de Zurich et en collaboration avec la Clinique pédiatrique universitaire de Zurich, une procédure spécifique pour évaluer les besoins en pédagogie spécialisée au niveau scolaire et postscolaire ${ }^{6}$.

La philosophie du SPZ comprend une collaboration étroite interdisciplinaire et multiprofessionnelle, regroupant les compétences des différentes spécialités sous un toit, ce qui rend possible des perspectives différenciées et des options diagnostiques et thérapeutiques spécifiques. Outre un réseautage interne intense, a lieu si nécessaire, une étroite collaboration avec les cabinets médicaux, les écoles ainsi que les institutions psychosociales de la région de Winterthour et des cantons voisins. Dans le cadre d'une évaluation globale est établi un profil individuel des ressources, troubles du développement et éventuels troubles psychiques, en fonction de l'âge des enfants et adolescents. Ils bénéficient d'une stimulation adaptée à leur possibilités et/ou d'un traitement. Les signes de conditions de développement défavorables, de stress accru et de charges liées à l'environnement sont discutés avec les familles afin de pouvoir offrir un soutien à un stade précoce ${ }^{7 / 8)}$. II existe une coopération étroite avec l'«lnstitut Kinderseele Schweiz» (iks), qui s'engage en faveur de l'égalité des chances de développement pour les enfants et les jeunes ayant un parent malade mental.

(Voir mon article dans ce numéro: enfants et jeunes issus de famille dont un parent souffre de troubles mentaux)

Le SPZ a une conception intégrée et globale du développement de l'enfant et de l'adolescent et s'oriente au modèle soi-disant étendu biopsychosocial ${ }^{9-11)}$.

La tranche d'âge prise en charge dans le domaine ambulatoire va de 0 à 18 ans. Les hospitalisations se font avec une approche parents-enfant pour les enfants d'env. 0-3 ans avec des troubles de la régulation, et pour des enfants d'env. 5-18 ans avec des troubles psychiques ou psychosomatiques. En milieu hospitalier le traitement des enfants et adolescents est multimodal, par des approches basées sur l'évidence et reconnues (approche orientée vers les solutions, thérapies systémiques, familiales, cognitivo-comportementales, schémathérapie, thérapie des États de Moi (Ego State), thérapie d'acceptation et d'engagement (ACT), etc.); ils fréquentent l'école de la clinique. La démarche est mentionnée depuis 2012 dans la liste des hôpitaux du Canton de Zurich. Sur mandat de la Ville de Winterthour, le SPZ assume par ailleurs le rôle de service de santé scolaire pour les presque 11'600 élèves des 588 classes d'école (enfantine) (d'après l'Office cantonal de l'enseignement obligatoire du Canton de Zurich pour 2020/2021).

Le SPZ est reconnu (par l'ISFM) en tant que centre de formation postgraduée pour les spécialités/formations approfondies suivantes:

\footnotetext{
- Psychiatrie et psychothérapie de l'enfant et adolescent*: 3 ans
}

- Pédiatrie du développement*: 2 ans 


\section{Formation continue}

- Neuropédiatrie*: 1 an

- Psychologie clinique

- Possibilités de stages: psychologie, pédagogie sociale, orthophonie

(Tableau 1)

\section{MEME, santé Mentale Enfant-adolescent Migrant et Ethnopsychanalyse}

\section{Un dispositif transverse d'évaluation et de soins} psychiques pour les réfugiés

Saskia von Overbeck Ottino, Psychiatre pour adultes et enfants-adolescents, ethnopsychanalyste, médecin associé HUG, vonoverbeckottino@bluewin.ch

En 2015, un nombre accru de réfugiés gagnait l'Europe, fuyant les conflits, essentiellement en Érythrée, en Afghanistan, en Irak et en Syrie. A Genève en particulier, malgré des bilans de santé somatique systématiquement proposés, il n'y avait pas d'augmentation des demandes en psychiatrie. Et pourtant, la littérature scientifique décrit unanimement un cumul de facteurs de risque affectant la santé psychique des réfugiés. Des facteurs pré-, per- et post- migratoires faits de traumatismes extrêmes au pays, de violences sur la route de l'exil et de précarités dans le pays d'accueil, sont autant de déterminants sociaux de la maladie psychique. Les statistiques à ce sujet font état de cinq fois plus de dépressions du post-partum, d'une augmentation significative des troubles de développement chez les enfants et, de manière générale, de troubles psychiatriques chez plus de $60 \%$ des réfugiés (Trouble Stress Post-Traumatique TSPT, dépression, troubles anxieux et abus de substances essentiellement) ${ }^{1)}$. Ce décalage entre des besoins évidents et le faible taux de demandes de soins psychiatriques, révélateur de difficultés de dépistage et d'accès aux soins, fut alors identifié comme un problème de santé publique et à l'origine de la création, au sein des Hôpitaux Universitaires de Genève, d'un dispositif dédié à faciliter l'accès aux soins en santé mentale pour les requérants d'asile: le dispositif MEME, santé Mentale Enfants-adolescents Migrants et Ethnopsychanalyse. L'appellation MEME, en plus de sa référence à l'autre semblable, fait écho au concept proposé par Richard Dawkins dans « Le Gène Égoïste » de mème (Dawkins, 1976). Un mème, par analogie à un gène, est un réplicateur non génétique, responsable de la transmission de comportements ou d'éléments culturels, permettant à un groupe social donné d'évoluer, un peu comme évoluent les êtres humains. Cette polysémie du nom MEME reflète bien l'esprit de notre dispositif, qui se veut à la fois rencontre avec le semblable, ouverture à la différence et espace d'évolution.

II s'agit d'une petite structure, 2 EPT (emploi plein temps), composé d'un psychiatre, de trois psychologues et d'une assistante sociale (AS), qui cible trois groupes à haut risque: les femmes/parents en période périnatale, les familles avec petits enfants et les mineurs non accompagnés. Nos interventions se font en collaboration étroite avec les médecins traitants en charge des bilans de santé.

Les mineurs non accompagnés (RMNA) sont systématiquement évalués dans les quelques semaines après leur arrivée à Genève. Ceci a permis d'améliorer l'identification de leurs difficultés, l'alliance thérapeutique de même que la collaboration avec l'ensemble du réseau et ses ressources. Nous nous sommes aperçus que $80 \%$ des RMNA présentaient des symptômes psychiatriques nécessitant un suivi prolongé ainsi qu'un soutien social individualisé. Concernant la périnatalité et les familles avec petits enfants, les suivis se font généralement en binômes, assistante sociale et psychologue, où l'AS propose un accompagnement «psycho-social» à domicile autour des aspects particuliers de la vie quotidienne d'un réfugié, et la psychologue des interventions psychothérapiques. Le médecin-cadre assure les synthèses du réseau, les entretiens de bilan, de couple ou de famille.

\begin{tabular}{|c|c|c|}
\hline Services & $\begin{array}{l}\text { Unités spécialisées } \\
\text { (ambulatoires) }\end{array}$ & $\begin{array}{l}\text { Consultations } \\
\text { (ambulatoires) }\end{array}$ \\
\hline $\begin{array}{l}\text { Hospitalisation } \\
\text { - Psychiatrie, psychosomatique et psychothérapie } \\
\text { - Approche parents-enfant (pour les troubles } \\
\text { de la régulation) } \\
\text { - Service de conseil et de liaison pédiatrie / urgences } \\
\text { Ambulatoire } \\
\text { - Psychiatrie, psychosomatique et psychothérapie } \\
\text { de l'enfant et adolescent } \\
\text { - Pédiatrie du développement } \\
\text { - Neuropédiatrie } \\
\text { - Service de santé scolaire de la Ville de Winterthour } \\
\text { - Recherche }\end{array}$ & $\begin{array}{l}\text { - Pédagogie spécialisée: ortho- } \\
\text { phonie (domaine pré- et post- } \\
\text { scolaire), pédagogie curative, } \\
\text { pédo-audiologie } \\
\text { - Autisme } \\
\text { - Expertise, conseil, thérapie }\end{array}$ & $\begin{array}{l}\text { - Psychotraumatologie } \\
\text { - Neuropsychologie } \\
\text { - Troubles alimentaires } \\
\text { - Troubles de la régulation } \\
\text { du nourrisson/petit enfant }\end{array}$ \\
\hline
\end{tabular}

\section{Tableau 1.}

Site web: https://www.ksw.ch/klinik/sozialpaediatrisches-zentrum-spz/ 


\section{Formation continue}

\section{3. «Et ta santé?»}

\section{Aller vers les populations \\ allophones et vulnérables. \\ Un projet d'accueil et d'évaluation de santé auprès des jeunes migrants scolarisés en Ville de Fribourg}

Dr Tina Huber-Gieseke, Médecine scolaire/ Service des écoles de la Ville de Fribourg, tina.huber-gieseke@ville-fr.ch

Vignette (2016): Mohamed, garçon de 14 ans originaire d'Erythrée, arrive en Suisse pour retrouver des membres de sa famille. Depuis la frontière, il est directement envoyé à Fribourg et scolarisé dans une classe d'accueil. Il n'a eu aucun contact médical depuis le début de son parcours migratoire. Il se présente à la visite médicale avec des douleurs abdominales chroniques.

Vignette (2019): Mère venue des Balkans avec ses 3 garçons en bas âge, alors que le père est resté au pays. Ils vivent dans une situation précaire. Durant les premiers mois, la mère n'inscrit pas ses 2 garçons de 5 et 7 ans à l'école par manque d'informations. Les autorités sont alertées et s'impliquent alors dans la situation lorsque le plus jeune fils est trouvé errant près de leur domicile. Une fois les deux aînés scolarisés, le médecin scolaire est appelé pour faire un bilan de santé.

En 2014-2015, de nombreuses familles migrantes, parfois avec de jeunes enfants, ainsi que des mineurs non accompagnés (MNA) sont arrivés à Fribourg. Leur état de santé, tant physique que psychique, était le plus souvent précaire, en raison des difficultés rencontrées dans leur parcours migratoire et de l'absence de prestations médicales depuis leur départ. De plus, l'arrivée dans une ville d'accueil est souvent une source de stress, mécanisme également observé en France voisine ${ }^{2}$. Ce constat nous a incité à mettre en place un projet interdisciplinaire agréé par la Direc- tion du Service des écoles (Partenaires: Secteur de Contact École-Parents-Migrants) (SCEPM), la médecine scolaire (MS), le Service des Écoles, Caritas «se comprendre» avec les interprètes communautaires).

II se base sur le postulat que l'école est un droit universel et l'accès à la santé un droit fondamental en Suisse ${ }^{3)}$. En collaboration avec le SCEPM (Secteur de Contact École-Parents-Migrants) dont la mission est d'accueillir l'ensemble des familles primo-arrivantes, une rencontre avec la médecine scolaire (MS) leur est proposée. L'objectif est d'offrir une évaluation de santé globale aux enfants et aux MNA scolarisés dans toutes les écoles de la ville de Fribourg. Une fois vu par l'infirmière et le médecin - la plupart du temps en présence d'un interprète communautaire - l'enfant ou le jeune est orienté vers un médecin privé ou un service adapté à sa problématique.

Une approche somatique et une anamnèse des habitudes de vie qui respecte la culture et les traditions offre souvent une porte d'entrée pour parler de soi et de ses préoccupations. Le stress est souvent omniprésent depuis leur arrivée chez l'enfant traumatisé mais aussi chez le parent isolé en attente d'un statut officiel et déjà préoccupé par sa propre intégration. II est un facteur de risque pour les maladies somatiques et psychiques ${ }^{11}$. C'est à travers une écoute professionnelle, qui repose sur un regard bienveillant, axée sur la santé qu'on parvient à diminuer cette pression. (Tableau 2)

Nous avons fait les observations suivantes:

- Un tiers des jeunes nécessite une orientation vers d'autres services d'aide (eg. Caritas).

- La présence d'un interprète communautaire permet d'intégrer les facteurs culturels, les traditions et les rituels, dont il faut tenir compte pour fonctionner efficacement en préservant un respect mutuel.

\begin{tabular}{|c|c|}
\hline Partenaires & En quoi consiste ce projet? \\
\hline SCEPM & $\begin{array}{l}\text { Suite à l'inscription à l'école, l'enfant et ses parents, souvent avec la fratrie, } \\
\text { sont adressés au SCEPM pour un premier accueil. La situation de santé est alors } \\
\text { abordée et s'ils le souhaitent, la famille ou le jeune sont mis en contact avec la MS. }\end{array}$ \\
\hline Infirmière scolaire & $\begin{array}{l}\text { L'infirmière scolaire procède à une anamnèse globale, pratique des tests } \\
\text { sensoriels, fait un relevé des paramètres généraux et des rappels de vaccins } \\
\text { et aborde les difficultés de santé. }\end{array}$ \\
\hline $\begin{array}{l}\text { Médecin scolaire } \\
\text { (MS) }\end{array}$ & $\begin{array}{l}\text { Le médecin pratique un examen clinique général et oriente l'enfant ou l'adolescent } \\
\text { MNA vers des services spécialisés. Si nécessaire, il prend contact avec des collè- } \\
\text { gues afin de faciliter la suite de la prise en charge dans ces situations souvent } \\
\text { encore peu claires sur le plan administratif. }\end{array}$ \\
\hline Réseau & $\begin{array}{l}\text { Parallèlement, le MS se met en contact avec les services d'aide à l'intégration } \\
\text { comme l'ORS pour les requérants d'asile et CARITAS pour les réfugiés. Au besoin, } \\
\text { une suite de prise en charge peut être organisée. }\end{array}$ \\
\hline $\begin{array}{l}\text { Directions } \\
\text { des écoles }\end{array}$ & $\begin{array}{l}\text { La direction des écoles informe le SCEPM de l'arrivée d'un enfant primo-arrivant } \\
\text { (EPA) et organise la venue d'un interprète si nécessaire. }\end{array}$ \\
\hline
\end{tabular}

Tableau 2. 


\section{Formation continue}

- Une collaboration étroite avec l'enseignant contribue à apaiser ses inquiétudes.

- Le SCEPM est une ressource précieuse pour les parents et pour les écoles (entretien d'accueil et de primo-information, accompagnement, coordination des bilans de compétences, collaborations avec d'autres partenaires autour du domaine de la migration).

Durant l'année COVID 19, ce projet a été adapté pour «aller vers les familles vulnérables», en offrant des consultations téléphoniques. Nous avons ainsi contacté 94 enfants de 58 familles durant la fermeture des écoles en 2020.

\section{Conclusion}

Pour certains, ces premières évaluations initient le processus d'intégration à l'école et mettent l'accent sur la santé globale. Notre suivi repose sur l'écoute et l'expression du corps et permet d'offrir une évaluation clinique gratuite, un rappel des vaccins, un contrôle de croissance et une orientation auprès des services d'intégrations et d'aide sur place. L'accès aux soins et l'accompagnement personnalisé, la possibilité de s'exprimer dans sa langue maternelle, ainsi que la scolarisation en double intégration sont ici des facteurs protecteurs d'une dégradation sur le plan psychique ou mental. Cette approche interdisciplinaire permet aux professionnels de travailler ensemble tout en préservant le secret médical et ainsi, de mieux accompagner les familles et jeunes migrants.

Des secteurs comme le SCEPM et la MS permettent de créer des ponts entre le monde de «là-bas» et celui d'«ici».

\section{En route ensemble - maladie chronique et école \\ Une approche du Service de santé scolaire de la Ville de Zurich}

Dr Dorothea Schultz, Directrice du projet «Zäme unterwegs - chronische Erkrankung und Schule», Schulgesundheitsdienste Stadt Zürich, Schulärztlicher Dienst, Zürich, dorothea.schultz@zuerich.ch

En 2013 on s'est rendu compte en ville de Zurich qu'à l'école publique la prise en charge d'enfants avec des besoins médicaux spécifiques était, dans des situations d'urgence, souvent mal coordonnée et que les cadres légaux n'étaient pas clairs. Un projet a donc été créé sous la conduite du Service de santé scolaire, en collaboration avec des parents, médecins et professionnels de l'école ainsi que la direction des écoles, afin de clarifier les bases légales et définir des procédures cohérentes. Suite à la décision du 9 juillet 2019 de la Commission scolaire de la Ville de Zurich toutes les écoles obligatoires de la ville disposent de l'offre «en route ensemble - maladie chronique et école» ${ }^{4)}$.
En substance la réglementation souhaite créer, par une information et une discussion précoce et transparente des besoins spécifiques, des conditions favorables pour que les enfants et adolescents avec des besoins médicaux particuliers puissent participer à autant d'activités scolaires que possible, et soient soutenus et pris en charge de manière compétente dans le cadre scolaire.

Il est donc décisif que les besoins médicaux et les mesures à prendre soient clairement définis tant du point de vue de l'enfant/adolescent concerné et de ses parents que de l'école (qui a pour mission le devoir d'instruction, de garde et de surveillance), et de trouver un dénominateur commun dans la mesure de la proportionnalité, de l'acceptabilité et de l'effectivement possible. Le soutien réciproque est fondamental. Au médecins scolaires incombe l'importante responsabilité de servir d'intermédiaires, d'expliquer les questions médicales de manière compréhensible au profane et de soutenir le cas échéant les parents, enfin de former les enseignants dans la gestion des urgences. Avec l'accord des parents ils collaborent étroitement avec les médecins traitants ${ }^{5}$.

\section{Et actuellement?}

Les parents concernés et les écoles recourent à cette offre. Les données de l'année en cours montrent que chez 823 (2.3\%) élèves de l'école obligatoire est connue une maladie chronique. Pour la plupart des élèves les répercussions des besoins médicaux sur le quotidien à l'école sont gérables et peuvent être pris en charge par l'enseignant. À titre d'exemple soit mentionné l'enfant avec un rhume des foins, qui dort moins bien pendant la saison pollinique et se fait remarquer à l'école par son manque de concentration. Les parents de cet enfant et l'enseignant peuvent préciser les besoins et les éventuelles mesures à prendre. Pour 195 élèves (0.5\%) nécessitant des secours spécifiques dans une situation d'urgence, une trousse d'urgence est déposée à l'école. Dans la plupart des cas il s'agit d'élèves allergiques avec un risque élevé de réaction anaphylactique, mais aussi d'élèves épileptiques, diabétiques, avec un asthme bronchique sévère, une maladie cardiaque ou d'autres maladies rares mais significatives. Lorsque des accidents potentiellement mortels peuvent survenir à l'école, tout le système entourant l'enfant, parents, médecin traitant et école, est sollicité afin de prévenir de telles situations ou agir correctement si l'urgence se présente. La communication ciblée des besoins actuels et spécifiques de l'élève à l'interne de l'équipe scolaire est dans ces cas élémentaire. L'instruction du corps enseignant concernant la manière de procéder en cas d'urgence (p.ex. instructions sur l'anaphylaxie) est essentielle.

\section{Combien d'écoles sont concernées?}

Statistiquement chaque école pourrait compter un enfant/adolescent nécessitant une prise en charge urgente spécifique. La répartition effective dans les écoles présente une tout autre réalité. Ainsi dans la salle des maîtres d'une école de taille moyenne de la ville de Zurich sont affichées les fiches d'urgence de 12 enfants, avec leur photo, afin que tous les ensei- 


\section{Formation continue}

gnants, chargés p.ex. de la surveillance pendant les récréations, aient un aperçu en un lieu central. Afin de pouvoir agir rapidement, les trousses d'urgence nominatives se trouvent à proximité et sont facilement accessibles. Plus les plans d'urgence sont uniformes et cohérents, plus il est facile pour les enseignants d'avoir rapidement une vue d'ensemble.

Toutes les personnes concernées apprécient l'engagement commun des professionnels et sont «en route ensemble» pour soutenir l'école dans l'effort de fournir aux élèves avec une maladie chronique, par des procédés simples et standardisées, un enseignement de qualité.

Pour la bibliographie, veuillez consulter notre version en ligne de l'article.

\section{Auteurs}

Dr. med. Kurt Albermann, Sozialpädiatrisches Zentrum SPZ, Departement Kinder- und Jugendmedizin, Kantonsspital Winterthur

Dr med. Saskia von Overbeck Ottino, psychiatre pour adultes et enfants-adolescents, ethnopsychanalyste, médecin associé HUG, Genève

Dr med Tina Huber-Gieseke, médecine scolaire/service des écoles de la Ville de Fribourg

Dr. med. Dorothea Schultz, Leitung Angebot «Zäme unterwegs - chronische Erkrankung und Schule», Schulgesundheitsdienste Stadt Zürich

Les auteurs n'ont déclaré aucun lien financier ou personnel en rapport avec cet article. 